\title{
Pulmonary embolism in a pregnant woman with COVID-19 infection: a case report
}

Sogand Goudarzi ${ }^{1}$, Fatemeh Dehghani Firouzabadi ${ }^{2}$, Fatemeh Mahmoudzadeh ${ }^{3}$, and Soheila Aminimoghaddam ${ }^{2}$

${ }^{1}$ Harvard Medical School

${ }^{2}$ Iran University of Medical Sciences

${ }^{3}$ Tehran University of Medical Sciences

September 16, 2020

\begin{abstract}
Coronavirus can lead to overcoagulation, blood stasis, and endothelial damage resulting in thromboembolic disorders. We report a 22-year-old pregnant woman with coronavirus admitted due to the pulmonary emboli. This case highlights the importance of considering a new category for COVID-19 pregnant patients with venous and arterial thromboembolic disorders.
\end{abstract}

\section{TITLE: Pulmonary embolism in a pregnant woman with COVID-19 infection: a case report}

Sogand Goudarzi, M.D. ${ }^{1}$; Fatemeh Dehghani Firouzabadi, M.D. ${ }^{2}$; Fatemeh Mahmoudzadeh, M.D ${ }^{3}$; Soheila Aminimoghaddam, M.D..$^{*}$

${ }^{1}$ Division of Cardiovascular Medicine, Department of Medicine, Beth Israel Deaconess Medical Center, Harvard Medical School, Boston, MA, United States

${ }^{2}$ ENT and Head and Neck Research Center and Department, Five Senses Health Research Institute, Hazrat Rasoul Akram Hospital, Iran University of Medical Sciences, Tehran, Iran

${ }^{3}$ Department of Emergency Medicine, Tehran University of Medical Sciences, Tehran, Iran

${ }^{4}$ Department of Gynecology Oncology, Iran University of Medical Sciences, Tehran, Iran

ORCID:

*Corresponding author : Dr. Soheila Aminimoghaddam

Address: Firoozgar Hospital, Iran University of Medical Sciences, Tehran, Iran

Tel: +982122211688

Email: Aminimoghaddam.s@iums.ac.ir

ORCID:

Sogand Goudarzi: 0000-0001-9552-2511

Fatemeh Dehghani Firouzabadi: 0000-0002-2665-3910

Soheila Aminimoghaddam: 0000-0001-6988-5722 
Word Count: 1154

Table Count: 1

Figure Count: 1

Conflict of Interest: Authors of this manuscript declare no conflict of interest of any nature.

Running Head: Pulmonary embolism in a COVID-19 pregnant female patient

\begin{abstract}
Coronavirus can lead to overcoagulation, blood stasis, and endothelial damage resulting in thromboembolic disorders. We report a 22-year-old pregnant woman with coronavirus admitted due to the pulmonary emboli. This case highlights the importance of considering a new category for COVID-19 pregnant patients with venous and arterial thromboembolic disorders.
\end{abstract}

Keywords: pregnancy, thrombosis, COVID-19, pulmonary embolism, case report, coagulopathy

\title{
Key clinical message
}

COVID-19 pregnant patients with venous and arterial thromboembolic disorders should be studied and treated in a separate category.

\section{Introduction}

Ever since the first case of coronavirus disease 2019 (COVID-19) in Wuhan, China, the world has been struggling to overcome this crisis. The rapid spread of the underlying severe acute respiratory syndrome coronavirus 2 (SARS-CoV-2) around the world, and its various complications imposed on the human body (which are not completely understood yet), have made the World Health Organization (WHO) declare a pandemic on March 11, 2020\{Cucinotta, 2020 \#22;Firouzabadi, 2020 \#816\}. Common symptoms of COVID-19 include but are not limited to dry cough, chest pain, shortness of breath, dyspnea, pneumonia, fever, fatigue, and in some cases death ${ }^{1-3}$. In addition to respiratory symptoms, COVID-19 can cause multiorgan disorders, the mechanism of which includes the release of inflammatory cytokines that stimulate tissue production and active thrombin ${ }^{4}$. Anticoagulant treatments are recommended for non-pregnant COVID-19 patients ${ }^{5}$.

Pregnant patients who are diagnosed with COVID-19 and show severe symptoms have a higher risk of thromboembolic disorders and can be treated with prophylactic weight-adjusted doses of heparin ${ }^{6}$. This study aims to introduce the uncommon manifestation of COVID-19 in pregnancy and its rarity, as wells as the more common thrombosis and DIC without any bleeding.

\section{Case Presentation:}

On April 22, 2020, a 22-year-old pregnant female with no past medical history and one-time previous natural delivery (gravid 2 para 1 live 1), with a gestational age of 30 weeks and 5 days was admitted to the emergency ward at Firoozgar Hospital, Tehran, Iran due to the loss of consciousness and double mydriasis. According to the patient's spouse, the patient has shown tonic-clonic seizure at home followed by loss of consciousness. Six days before admission, the patient had presented shortness of breath for several days what she consumed inhaled opioids, which she declared that she did not have an addiction before.

In the emergency room, the patient was intubated due to the loss of consciousness and a low score on the Glasgow Coma Scale. Cardiopulmonary resuscitation (CPR) was performed on her. The fetal heartbeat was not detected. After consulting with the anesthesiologist and the cardiologist, the patient was then quickly transferred to the operation room for monitoring and possible cesarean delivery. The pregnancy was 
terminated prematurely due to not detecting the fetal heartbeat and saving the mother's life because of the unstable condition leading to eight rounds of CPR. The CPR on the patient was performed with 2 doses atropine (2 mg intravenously), 2 vials calcium gluconate, 5 vials sodium bicarbonate, and 10 intravenous vials of epinephrine $(10 \mathrm{mg})$. Emergency echocardiography in the operating room was performed, which showed a very dilated right atria and ventricle, leading to the full pressure of the intercostal wall on the left ventricle. The pulmonary artery pressure was measured to be 50 and ejection fraction (EF) was $30 \%$, resulting in a diagnose of a massive pulmonary embolism and the right- and left-sided heart failure (additional echocardiography results are as follows: right ventricle enlargement, severe dysfunction McConnell Sign, moderate tricuspid regurgitation and no tricuspid stenosis, systolic pulmonary pressure (sPAP) of 35, dilated pulmonary artery, mild pulmonary insufficiency and no pulmonic stenosis, no aortic insufficiency and aortic stenosis, no mitral regurgitation and mitral stenosis, dilated inferior vena cava, andq normal left ventricle size). An intravenous single dose (100 mg) alteplase was immediately infused due to the critical condition of the mother with the very low EF, and the fetal death in the mother's uterus confirmed with ultrasound. In consultation with a cardiologist, they offered to do embolectomy, but it was not possible at this center. Also, the patient was not at a stable stage to be transferred to another place. So, alteplase was started.

The patient was transferred to the Intensive Care Unit (ICU) when she became stable. She was treated with $3 \mathrm{mg}$ of Midazolam injection (intravenously if necessary), $500 \mathrm{mg}$ Levebel injection (intravenously twice a day), $1 \mathrm{mg}$ intravenous injection of cefepime twice a day, $25 \mu \mathrm{g}$ of Fentanyl injection (intravenously as needed), daily intravenous injection of $40 \mathrm{mg}$ Pantoprazole, $40 \mu \mathrm{g} / \mathrm{min}$ of norepinephrine infusions, $3-5 \mu \mathrm{g} / \mathrm{hr}$ of midazolam infusions, $25-50 \mu \mathrm{g} / \mathrm{hr}$ of fentanyl infusions, and one intravenous vial of bicarbonate for $\mathrm{pH}$ levels lower than 7.2.Table 1 shows the results of the lab reports, which confirmed that the patient was tested positive COVID-19. Chest X-Ray also confirmed the same diagnosis, which demonstrated diffuse consolidative opacities in both lungs with the left side being predominant(Figure 1) .

The extra-amniotic saline infusion (EASI) was installed to end the pregnancy, the dilation was $5 \mathrm{~cm}$ while it was removed, and the patient expired before delivery. During ICU admission, despite receiving norepinephrine infusions, the patient's blood pressure was very low $(70 / 40)$ with the clubbed vascular resulting in putting a central venous line on her femur with extreme difficulty. The patient expired due to respiratorycardiovascular arrest and unsuccessful cardiopulmonary resuscitation on April 23, 2020

\section{Discussion}

COVID-19, which initially presents with symptoms of respiratory illness, may lead to dysfunction of a single organ or multiple organs and even death. In non-pregnant patients admitted to the ICU with COVID-19 pneumonia, the prevalence of venous and arterial thromboembolic disorders is reported to be about $25 \%$ to $31 \%^{7,8}$.

A recent study considered a new category for COVID-19 patients with venous and arterial thromboembolic disorders (named as COVID-19 associated coagulopathy) and compared it to other thromboembolic disorders such as disseminated intravascular coagulation, hemophagocytic syndrome, antiphospholipid syndrome, thrombotic microangiopathy, thrombotic thrombocytopenic purpura, and Heparin-induced thrombocytopenia ${ }^{9}$. Our patient had some parameters of COVID-19 associated coagulopathy such as high PTT, fibrinogen, and D-Dimer levels. Higher D-dimer levels (more than $0.5 \mu \mathrm{g} / \mathrm{mL}$ ) are considered as an indirect indicator for increased thrombin production and are associated with an increased risk of death ${ }^{10,11}$. Anticoagulant therapy with low molecular weight heparin (LMWH) shows promising results in the prognosis of severe COVID-19 patients with higher levels of D-dimer by limiting the extent of coagulopathy ${ }^{12}$.

Treatment by Heparin can also reduce the inflammatory biomarkers leading to a decline in the severity of COVID-19 infection ${ }^{13}$. According to a study by Betoule et al., preventive anticoagulant treatments should be considered in COVID1-19 non-pregnant patients with D-dimer [?] $3 \mu \mathrm{g} / \mathrm{ml}$ (11 mdf). Dashraath et al. determined that pregnant women suspected of the severe form of COVID-19 infection during the third trimester are at a higher risk of thromboembolic disorders. Therefore, they suggested that these pregnant 
women be given the prophylactic weight-adjusted dose of heparin during hospitalization, continued until delivery, and six weeks postpartum ${ }^{6}$.

Like ours, a report in Milan, Italy, presented a case of a 17 -year-old obese pregnant on $29^{\text {th }}$ week of pregnancy with shortness of breath lasted for a few days After initial assessment - she was diagnosed with pulmonary embolism at the hospital and was received immediately antithrombotic treatment before and after the delivery, which saved her from further complications ${ }^{14}$.

To our best knowledge, this is the first report of maternal death due to COVID-19 associated coagulopathy. As a high number of pregnant women (25 to 30\%) with MERS and SARS dead ${ }^{15}$, it is worthwhile to consider the maternal death in COVID-19 infection especially in the third trimester due to coagulative disorders that can be prevented via prophylactic treatment.

The result of this study could increase awareness and help the frontline worker or doctors to be well prepared to treat such patients promptly and hopefully, save lives.

\section{Acknowledgements}

We would like to thank Dr. Gerald Chi of the Cardiovascular Department at Harvard Medical School for his critical reviews and valuable opinions.

Table 1. Patient Laboratory Data

\begin{tabular}{|c|c|c|c|c|}
\hline April 23, 2020 & April 23, 2020 & April 22, 2020 & April 22, 2020 & Date \\
\hline 9:40 & 00:00 & $16: 55$ & $10: 40$ & Time \\
\hline 26.8 & 12.5 & 19 & 10.8 & WBC (per mm³) \\
\hline 9.6 & 20.5 & 32.8 & 60.3 & lymphocyte \\
\hline 3.15 & 3.55 & 3.63 & 3.47 & $\mathrm{RBC}\left(\right.$ per $\left.\mu \mathrm{m}^{3}\right)$ \\
\hline 9.5 & 11.1 & 11.2 & 10.5 & $\mathrm{Hb}(\mathrm{g} / \mathrm{dl})$ \\
\hline 29.1 & 33.5 & 34.6 & 33.9 & Hct (\%) \\
\hline 92.4 & 94.4 & 95.3 & 97.7 & MCV (fL) \\
\hline 30.2 & 31.3 & 30.9 & 30.3 & $\mathrm{MCH}(\mathrm{Pgm})$ \\
\hline 32.6 & 33.1 & 32.4 & 31 & $\mathrm{MCHC}(\mathrm{gr} / \mathrm{dL})$ \\
\hline 103 & 141 & 158 & 187 & Platelet Count $\left(\right.$ per $\left.\mathrm{mm}^{3}\right)+$ \\
\hline 69.1 & NA & $\max$ & $18 / 6$ & $\mathrm{PT}(\mathrm{Sec})$ \\
\hline 82 & NA & 132 & 46 & $\mathrm{PTT}(\mathrm{Sec})++$ \\
\hline 5.6 & NA & NA & 1.4 & INR \\
\hline NA & NA & NA & Negative & Antiphospholipid Antibody \\
\hline NA & NA & NA & Negative & Anticardiolipin Antibody \\
\hline NA & NA & NA & Negative & Lupus anticoagulant \\
\hline 7.05 & 7.03 & 6.6 & 6.4 & $\mathrm{pH}$ \\
\hline 41 & 42.7 & 211.6 & 172 & $\mathrm{pCO}_{2}(\mathrm{~mm} \mathrm{Hg})$ \\
\hline-18.6 & 19.2 & -21.9 & -34 & $\mathrm{BE}(\mathrm{mmol} / \mathrm{l})$ \\
\hline 11.5 & 11 & 40.8 & 15.1 & $\mathrm{HCO}_{3}(\mathrm{mmol} / \mathrm{l})$ \\
\hline 39 & 55.9 & 51.3 & 18.8 & $\mathrm{pO}_{2}(\mathrm{~mm} \mathrm{Hg})$ \\
\hline 111 & NA & 59 & 45 & BUN (mg/dl) \\
\hline 3.6 & NA & 1.6 & 1.4 & Creatinine $(\mathrm{mg} / \mathrm{dl})$ \\
\hline 139 & NA & 140 & 140 & $\mathrm{Na}$ \\
\hline 5.3 & NA & 4.7 & 4.4 & $\mathrm{~K}$ \\
\hline NA & NA & NA & 8.4 & $\mathrm{Ca}$ \\
\hline 2.2 & NA & NA & NA & $\mathrm{Mg}$ \\
\hline 6 & NA & NA & NA & $\mathrm{P}$ \\
\hline NA & NA & NA & Normal & Urine analysis \\
\hline
\end{tabular}




\begin{tabular}{lllll} 
NA & NA & NA & No growth & Urine culture \\
NA & NA & Staphylococci epidermidis & Blood culture \\
2000 & NA & 2200 & 2829 & SGOT(IU/L) \\
2058 & NA & 2100 & 2637 & SGPT(IU/L) \\
1218 & NA & 1038 & 939 & Alk-P \\
2.4 & NA & 1.4 & NA & Bilirubin-Total \\
1.2 & NA & 0.6 & NA & Bilirubin-Direct \\
NA & NA & NA & 2184 & CPK \\
NA & NA & NA & 6240 & LDH (per ml) \\
10.5 & NA & NA & NA & Beta2-microglobulin $(\mu \mathrm{g} / \mathrm{ml})$ \\
NA & NA & NA & Negative & HBsAg Antibody \\
NA & NA & NA & Negative & HIV Antibody \\
NA & NA & NA & +3 & D-dimer \\
NA & NA & NA & 81 & Fibrinogen $(m g / d l)+$ \\
NA & NA & NA & Positive & PCR COVID-19 \\
\hline
\end{tabular}

Time: testing time; WBC: white blood cells; RBC: red blood cells; Hb: hemoglobin; Hct: hematocrit; MCV: mean cell volume; $\mathrm{MCH}$ : mean cell hemoglobin; MCHC: mean cell hemoglobin concentration; PT: prothrombin time; PTT: partial thromboplastin time; INR: international normalized ratio; BE: base excess; BUN: blood urea nitrogen; Na: Sodium; K: Potassium; Ca: Calcium; Mg: Magnesium; P: Phosphorus; SGOT: serum glutamic-oxaloacetic transaminase; SGPT: serum glutamic-pyruvic transaminase; Alk-P: Alkaline phosphatase; CPK: Creatine phosphokinase; LDH: Lactate dehydrogenase; HBsAg: Hepatitis B surface antigen; HIV: human immunodeficiency viruses; PCR: polymerase chain reaction; COVID-19: coronavirus disease 2019; NA: Not available.

+: Decreased according to the laboratory normal range

++ : Prolonged 


\section{Figure legends}

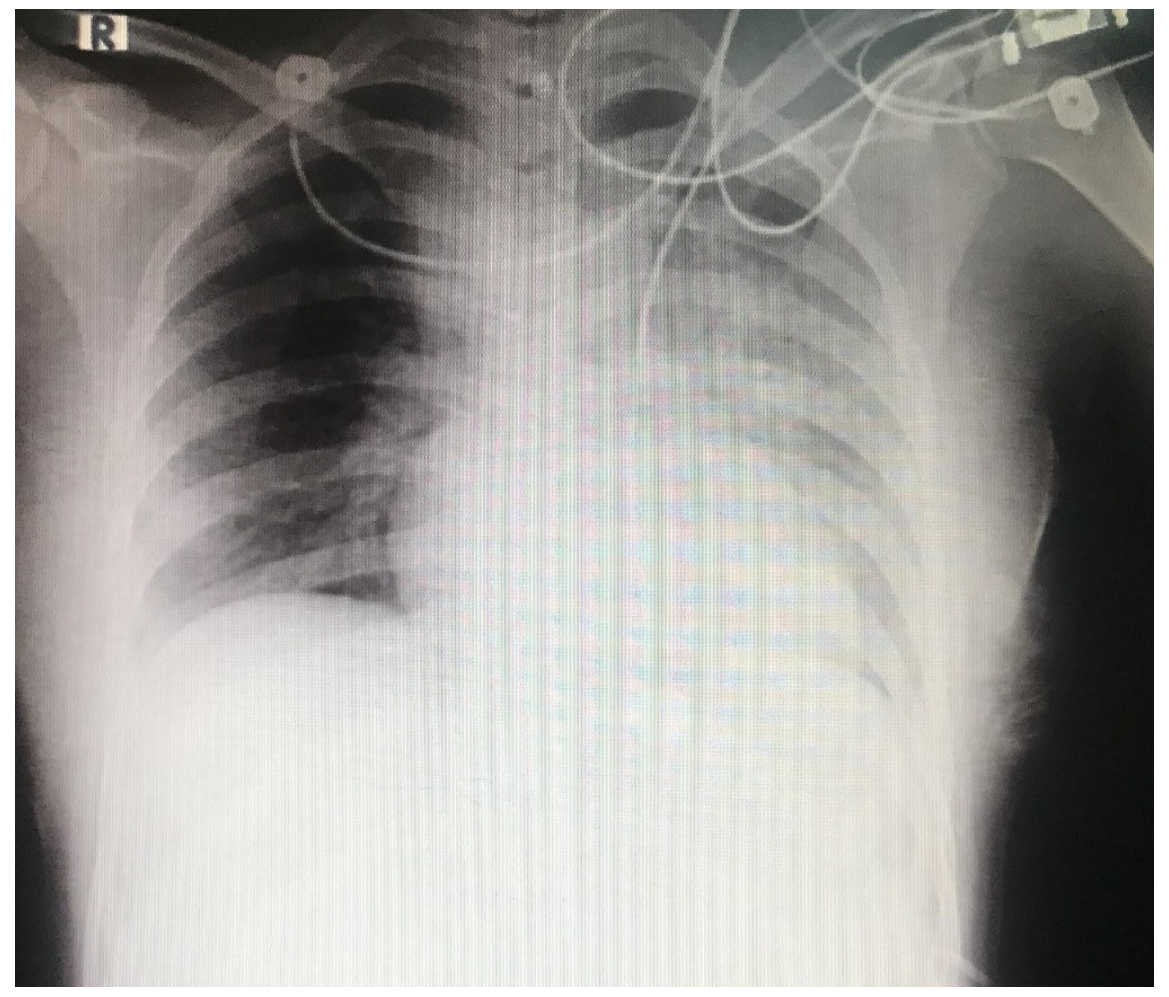

Figure 1. Patient chest X-Ray

\section{Author contributions}

Sogand Goudarzi: Introduction, case presentation, discussion, coordination, article authorship and editing Fatemeh Dehghani Firouzabadi: Data collection, part of discussion

Fatemeh Mahmoudzadeh, M.D: Data collection

Soheila Aminimoghaddam, M.D: Patient treatment, data collection, correspondence

\section{REFERENCES}

1. Dehghani Firouzabadi M, Dehghani Firouzabadi F, Goudarzi S, Jahandideh H, Roomiani M. Has the chief complaint of patients with COVID-19 disease changed over time? Medical Hypotheses.2020;144:109974.

2. Firouzabadi FD, Firouzabadi MD, Ghalehbaghi B, Jahandideh H, Roomiani M, Goudarzi S. Have the symptoms of patients with COVID-19 changed over time during hospitalization? Medical Hypotheses. 2020;143:110067.

3. Dehghani Firouzabadi F DFM, Ghalehbaghi B, Jahandideh H, Roomiani M, Goudarzi S, Moosaie F, Chi G. What is the difference between the first and second COVID-19 outbreak in Iran? International Journal of Cardiovascular Diseases 63 Diagnosis. 2020;In Press.

4. Qin C, Zhou L, Hu Z, et al. Dysregulation of Immune Response in Patients With Coronavirus 2019 (COVID-19) in Wuhan, China.Clinical Infectious Diseases. 2020. 
5. Betoule A, Martinet C, Gasperini G, et al. Diagnosis of venous and arterial thromboembolic events in COVID-19 virus-infected patients.J Thromb Thrombolysis. 2020;50(2):302-304.

6. Dashraath P, Wong JLJ, Lim MXK, et al. Coronavirus disease 2019 (COVID-19) pandemic and pregnancy. American Journal of Obstetrics and Gynecology. 2020;222(6):521-531.

7. Cui S, Chen S, Li X, Liu S, Wang F. Prevalence of venous thromboembolism in patients with severe novel coronavirus pneumonia. (1538-7836 (Electronic)).

8. Klok FA, Kruip MJHA, van der Meer NJM, et al. Incidence of thrombotic complications in critically ill ICU patients with COVID-19. Thromb Res. 2020;191:145-147.

9. Iba T, Levy JH, Connors JM, Warkentin TE, Thachil J, Levi M. The unique characteristics of COVID-19 coagulopathy. Critical Care.2020;24(1):360.

10. Zhou F, Yu T, Du R, et al. Clinical course and risk factors for mortality of adult inpatients with COVID-19 in Wuhan, China: a retrospective cohort study. The Lancet.2020;395(10229):1054-1062.

11. Dehghani Firouzabadi M, Goudarzi S, Dehghani Firouzabadi F, Moosaie F. Complete heart block and an itching rash in a patient with COVID-19. Caspian Journal of Internal Medicine. 2020.

12. Tang N, Bai H, Chen X, Gong J, Li D, Sun Z. Anticoagulant treatment is associated with decreased mortality in severe coronavirus disease 2019 patients with coagulopathy. (1538-7836 (Electronic)).

13. Whyte CS, Morrow GB, Mitchell JL, Chowdary P, Mutch NJ. Fibrinolytic abnormalities in acute respiratory distress syndrome (ARDS) and versatility of thrombolytic drugs to treat COVID-19. Journal of Thrombosis and Haemostasis. 2020;18(7):1548-1555.

14. Martinelli I, Ferrazzi E, Ciavarella A, et al. Pulmonary embolism in a young pregnant woman with COVID-19. Thromb Res. 2020;191:36-37.

15. Di Mascio D, Khalil A, G S. Outcome of Coronavirus spectrum infections (SARS, MERS, COVID 1 -19) during pregnancy: a systematic review and meta-analysis. Am J Obstet Gynecol 2020;2(2):100-107. 


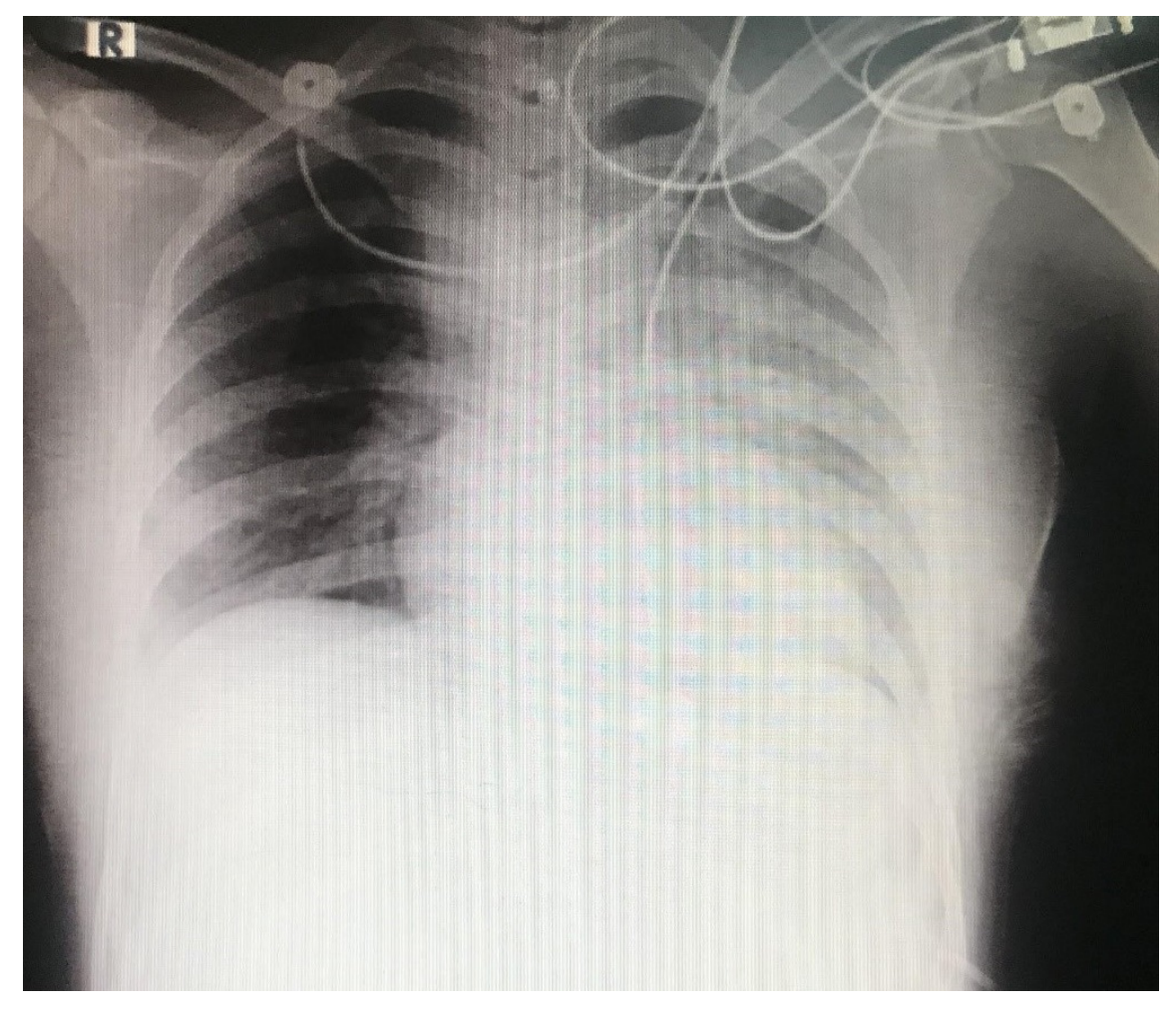

